\title{
KINEMATICS OF ABELL 30
}

\author{
George H. Jacoby \\ Kitt Peak National Observatory \\ You-Hua Chu \\ Astronomy Department, University of Illinois
}

Abe11 30 is a remarkable PN with a H-depleted core. Four bright knots have been identified in the [O III] line (Jacoby 1979). The previous kinematic study has shown that $\mathrm{J} 1$ and $\mathrm{J} 3$ form a pair expanding at radial velocities of $\pm 25 \mathrm{~km} / \mathrm{s}$ with respect to the central star, and $\mathrm{J} 4$ has a radial velocity of $-22 \mathrm{~km} / \mathrm{s}$ (Reay, Atherton, and Taylor 1983).

We have obtained deep-exposure echelle spectra of the knots in the core of $\mathrm{A} 30$. The results, while confirming the radial velocities of $\mathrm{J} 1$ and J3, show previously-undetected multiple components in J2 and J4.

The average heliocentric velocity of $A 30$ is $+10 \mathrm{~km} / \mathrm{s}$. The knot J1 is dominated by one single component at $+34 \mathrm{~km} / \mathrm{s}$, while $\mathrm{J} 3$ is dominated by a single component at $-13 \mathrm{~km} / \mathrm{s}$. The knot $\mathrm{J} 2$ is kinematically resolved into at least four components at velocities of $-13,+31,+60$, and $+87 \mathrm{~km} / \mathrm{s}$, with the $+31 \mathrm{~km} / \mathrm{s}$ component being the brightest. The knot $\mathrm{J} 4$ is resolved into four components at $\geq+66,+36,-10$, and -50 $\mathrm{km} / \mathrm{s}$; the brightest component at $-10 \mathrm{~km} / \mathrm{s}$ appears tilted with $-2 \mathrm{~km} / \mathrm{s}$ at the inner edge and $-17 \mathrm{~km} / \mathrm{s}$ at the outer edge.

The components in $\mathrm{J} 2$ and $\mathrm{J} 4$ form arc-like patterns in the echellograms. Such pattern can be explained by a clumpy expanding ring structure. The position angle of the ring is similar to that of the infrared disk detected by Dinerstein and Lester (1984).

S. Torres-Peimbert (ed.), Planetary Nebulae, 183.

(c) 1989 by the IAU. 